\title{
Update on the management of refractory variceal bleeding
}

\author{
Kapil D Jamwal*, Rajesh K Padhan and Atul Sharma
}

Department of Gastroenterology, Artemis Hospital, Gurugram, Haryana, India

Acute variceal bleeding (AVB) is seen in $50-70 \%$ of patients with cirrhosis \& portal hypertension (PHT) [1]. Over the passage of time the severity of the variceal bleeding and complications related to it have significantly reduced. This reduction in complications is due to improvement in the clinical management, universal availability of vasopressor drugs, improved endoscopic therapies as well as due to availability of definitive treatment options such as trans jugular intra hepatic porto systemic shunt (TIPS) and liver transplantation. About $10-20 \%$ patients presenting with AVB do not respond to the initial management (defined as failure to control bleeding - within $48 \mathrm{hrs}$ ) and develop re bleeding within 5 days of starting the therapy (after initial control of bleeding), these patients can be defined to have refractory variceal bleeding (Figure 2).

The causes for refractory variceal bleeding are:

1. severe liver disease (high MELD-Na and CTP score)

2. coagulopathy (increased PT, INR and low platelets)

3. post EVL band ulcers (PEBU's),

4. slippage of bands or

5. presence of SRH (stigmata of recent hemorrhage - platelet plug, ulcer) or RCS (red color signs - red wale signs, cherry red spots) [2-4].

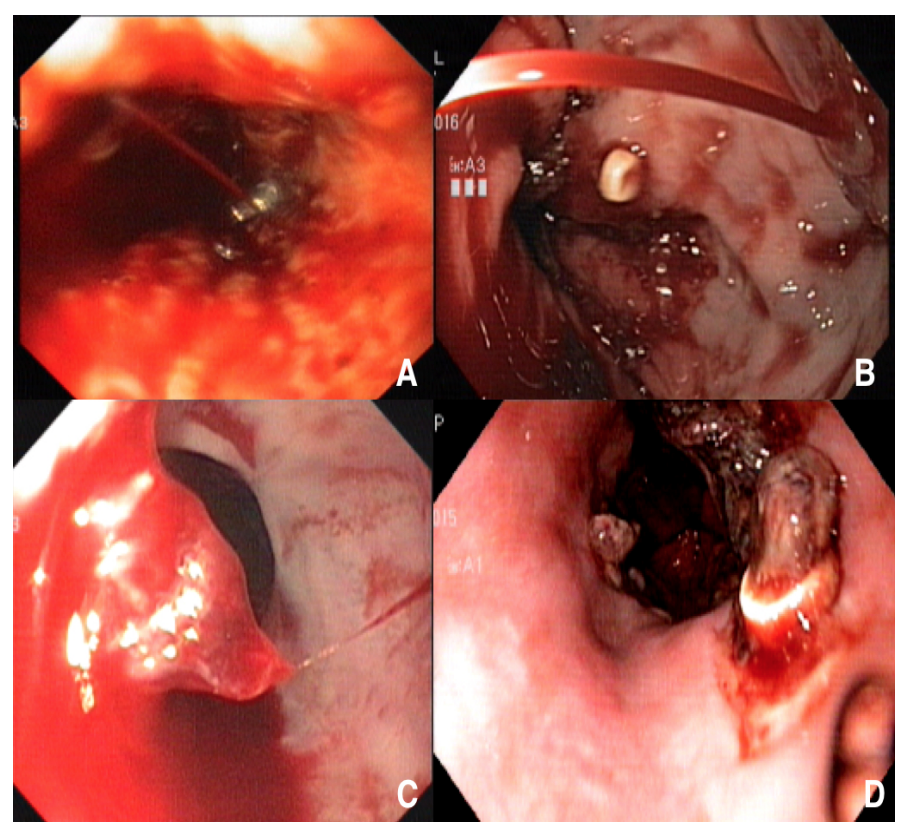

Figure 1. Shows refractory variceal bleeding in different endoscopic scenarios, a. spurting from the varices with EVL bands in situ, b. spurting from the varices after slippage of the EVL bands, c. ooze from the EVL band site, d. post band - clot formation and ooze from the base of the band site.

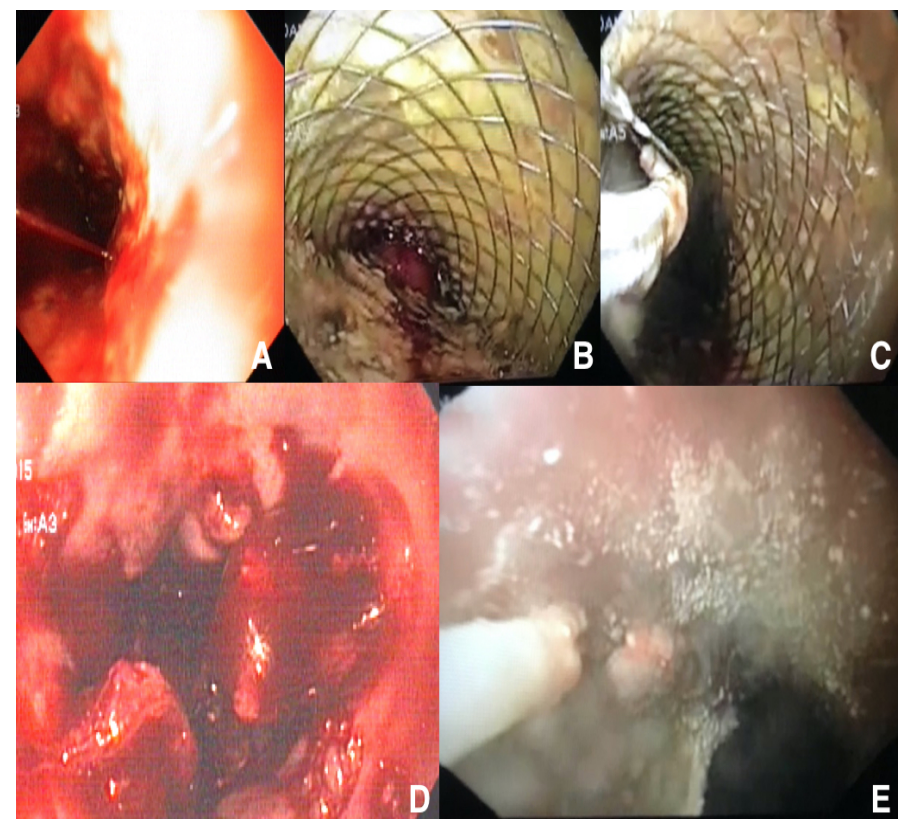

Figure 2. Shows management of refractory variceal bleeding, in images a,b and c: there is active bleeding(a) which is followed by placement of a SEMS, (b) and then SEMS removal, (c) in pictures, $d$ and e: show oozing from the varices (d) which was treated with hemospray application (e).

In case of treatment failure in an acute bleeding episode, if bleeding is mild and the patient is hemodynamically stable, a second endoscopic therapy should be attempted. If this fails, or if bleeding is severe, it is usually controlled temporarily with balloon tamponade until a definitive derivative treatment is applied. Despite all treatment, a significant proportion of patients do not respond to the therapy. Failure to control bleeding leads to poorer outcomes due to:

1. worsening liver failure

2. development of organ dysfunction due to progressive shock, organ failure

3. systemic sepsis and increased gut translocation of bacteria.

The management in this group of patients depends upon the general condition of the patient and the underlying liver disease status. There was a recent study which showed that endoscopic appearance of

*Correspondence to: Kapil D Jamwal, Department of Gastroenterology, Artemis Hospital, Gurugram, Haryana, India, Tel: +91-11-46300000, Fax: +91-114600025, E-mail: drkapil222@gmail.com

Received: August 10, 2020; Accepted: August 24, 2020; Published: August 31, 2020 
post banding ulcers can determine the patient outcomes in addition and independent to MELD-Na score [4]. It is a combined team effort involving a hepatology, diagnostic radiology, intervention radiology and a liver transplant team.

The goals of the treatment in refractory variceal bleeding is:

1. maintain hemodynamic stability \& tissue oxygenation.

2. control of bleeding

3. prevention of complications

\section{Generalized treatment for stabilization of the patient}

A. Blood Transfusion: In addition to fluid resuscitation, blood component replacement is an important part of resuscitation in AVB. This includes blood transfusion (packed red blood cell) to maintain a hemoglobin level of 7-8 gram/dl. The restrictive vs liberal treatment regimen of blood transfusion has shown that the restrictive strategy is better in terms of re bleeding and mortality in AVB [5].

B. Coagulopathy correction: The patients with coagulopathy can be treated either with blood products based on lab parameters (INR, platelet count, aPTT) or blood products based on TEG (thromboelastography). The conventional approach in coagulopathy with variceal bleeding is to administer blood components based on abnormalities detected during routine blood tests, but the data on this regimen does not support use of nonspecific blood products in AVB due to its associated side effects and complications.

A recent RCT was done in cirrhotic with GI bleeding and coagulopathy, in this study the patients presented with non-variceal GI bleeding and were managed with TEG guided correction in comparison to standard INR guided correction for coagulopathy. The study concluded that TEG based correction of coagulopathy showed significant reduction in number of blood products being transfused and it was not associated with increased complications, mortality, and bleeding [6,7].

C. Antibiotics: The use of antibiotics is recommended for 5-7 days in the management of variceal bleeding. Broad spectrum antibiotics is recommended in advanced liver disease and third generation cephalosporins are recommended in the compensated liver disease patients. Early administration of antibiotics after AVB has been associated with decreased incidence of rebleeding and overall morbidity [8].

D. Vasopressors: Systemic vasopressors have to be used in the initial management of AVB and a RCT suggested that there was no difference on the type of vasopressor used (terlipressin, octreotide or somatostatin). The vasopressors should be started as early as possible depending upon the availability of the drug. The APASL guidelines for variceal bleeding has advised to target a door to needle (arrival in hospital to the injection of vasopressors) time akin to strategy being used in the management of acute coronary syndromes [2,3,9] (Figure 1).

\section{Control of bleeding i.e. local or systemic}

\section{A. Local treatment:}

i) Tamponade: The tamponade is an effective method to achieve short term homeostasis, these therapies include balloon tamponade, using either a triple lumen (Sengstaken Blakemore tube), a four lumen (Minnesota) or a Linton Nicholas tube (single $600 \mathrm{ml}$ gastric balloon) for control of AVB, these measures are useful in the cases when there is failure to control bleeding at the initial endoscopy or in situations where emergency endoscopy is not available. A retrospective study showed that there was improvement of short-term survival (1 month) as well as long term (1 year) after application of local tamponade in AVB [10].

ii) Endoscopic treatment: This should be instituted as early as possible with a goal to perform endoscopy within 12 hours of AVB.

Local endoscopic therapies include injection of glue, sclerosants and reapplication of bands over the bleeding ulcer. These are used if the deployment of a band is difficult, such as in: a. very small varices, not amenable for banding $b$. a very large ulcer on which a band cannot be deployed c. excessive bleeding in which there is failure to localize the bleeding spot. In these conditions, injection therapies can be considered with refractory bleeding. (11) The short-term complications of the injection therapies are mediastinitis, bacteremia, ulcer formation and rebleeding.

iii) Hemospray application: In a recent RCT the patients with acute variceal bleeding (AVB) were randomized to standard of care (SOC) vs addition of hemospray to SOC, the study showed that the group in which hemospray was used in addition to SOC showed better outcomes and significantly reduced mortality as compared to SOC alone. However, it is worthy to note that the selection of cases was in first bleeding episode and no cases with refractory bleeding was included in the study. Hence the hemospray can be used as one of the local methods to control variceall bleeding [12].

iv) Placement of SEMS (self-expanding metal stents): There are ample studies available which demonstrate that by using SEMS specially designed for refractory variceal bleeding, the bleeding can be significantly controlled, and the outcomes improved in this patient group. Most of these studies have shown that the SEMS helps in the control of bleeding locally by tamponade over the bleeding varices, however placement of the SEMS has to followed by a definitive treatment procedure such TIPS for reduction of the portal pressure or a liver transplant as definitive treatment since the SEMS has to be removed within 1-2 weeks of deployment. A recent meta analysis showed the technical success to be $97 \%$ with control of bleeding in $96 \%$ [13-16].

\section{B. Systemic treatment:}

i) TIPS is a treatment for patients who present with refractory bleeding with early liver disease $($ MELD $<18)$. There is enough data now to suggest that TIPS is a SOC in refractory variceal bleeding. But in cases with hemodynamic instability, poor liver function (MELD-Na $>18$ ) or previous episodes of hepatic encephalopathy, doing TIPS is a relative contraindication $[17,18]$.

ii) Surgical options including liver transplantation: in patients who have refractory bleeding and are not candidates for TIPS due to advanced liver disease (MELD > 18), ACLF or prior history of recurrent hepatic encephalopathy. These patients can be taken for a liver transplantation as a definitive treatment [17-20].

In conclusion, refractory variceal bleeding is noted in $10-20 \%$ of AVB episodes. Failure to control bleeding leads to further complications and increased morbidity-mortality. Treatment in such patients should be individualized and depends upon the local set up and availability of options at the treating facility. 


\section{References}

1. D'Amico M, Berzigotti A, Garcia-Pagan JC, et al. (2010) Refractory acute variceal bleeding: what to do next? Clin Liver Dis 14: 297-305. [Crossref]

2. Sarin SK, Kumar A, Angus PW, Baijal SS, Baik SK, et al. (2011) Asian Pacific Association for the Study of the Liver (APASL) Working Party on Portal Hypertension. Diagnosis and management of acute variceal bleeding: Asian Pacific Association for Study of the Liver recommendations. Hepatol Int 5: 607-624. [Crossref]

3. Dev JK (2016) Modified APASL bleeding score predicts outcome better than other scores in ACLF: A prospective study from the multinational AARC consortium. $J$ Gastroenterol Hepatol 31: 111.

4. Jamwal KD, Maiwall R, Sharma MK, Kumar G, Sarin SK (2019) Case Control Study of Post-endoscopic Variceal Ligation Bleeding Ulcers in Severe Liver Disease: Outcomes and Management. J Clin Transl Hepatol 7: 32-39. [Crossref]

5. de Franchis R, Baveno VI (2015) Expanding consensus in portal hypertension: Report of the Baveno VI Consensus Workshop: Stratifying risk and individualizing care for portal hypertension. J Hepatol 63: 743-52.

6. Kumar M,Ahmad J, Maiwall R, ChoudhuryA, Bajpai M, etal. (2020) ThromboelastographyGuided Blood Component Use in Patients With Cirrhosis With Nonvariceal Bleeding: A Randomized Controlled Trial. Hepatology 71: 235-246. [Crossref]

7. Lisman T, Caldwell SH, Burroughs AK, Northup PG, Senzolo M, et al. Coagulation in Liver Disease Study Group. Hemostasis and thrombosis in patients with liver disease: the ups and downs. J Hepatol 53: 362-371. [Crossref]

8. Garcia-Tsao G, Abraldes JG, Berzigotti A, Bosch J (2017) Portal hypertensive bleeding in cirrhosis: Risk stratification, diagnosis, and management: 2016 practice guidance by the American Association for the study of liver diseases. Hepatology 65: 310-335. [Crossref]

9. Seo YS, Park SY, Kim MY, Kim JH, Park JY, et al. (2014) Lack of difference among terlipressin, somatostatin, and octreotide in the control of acute gastroesophageal variceal hemorrhage. Hepatology 60: 954-963. [Crossref]

10. Nadler J, Stankovic N, Uber A, Holmberg MJ, Sanchez LD, et al. (2017) Outcomes in variceal hemorrhage following the use of a balloon tamponade device. Am J Emerg Med 35: 1500-1502.
11. European Association for the Study of the Liver. Electronic address: easloffice@ easloffice.eu; European Association for the Study of the Liver (2018) EASL Clinical Practice Guidelines for the management of patients with decompensated cirrhosis. $J$ Hepatol 69: 406-460.

12. Ibrahim M, El-Mikkawy A, Abdel Hamid M, AbdallaI H, Lemmers A, et al. (2019) Early application of haemostatic powder added to standard management for oesophagogastric variceal bleeding: a randomised trial. Gut 68: 844-853. [Crossref]

13. Hernández-Gea V, Procopet B, Giráldez Á, Amitrano L, Villanueva C, et al. (2019) International Variceal Bleeding Observational Study Group and Baveno Cooperation. Preemptive-TIPS Improves Outcome in High-Risk Variceal Bleeding: An Observational Study. Hepatology 69: 282-293. [Crossref]

14. Maiwall R, Jamwal KD, Bhardwaj A, Bhadoria AS, Maras JS, et al. (2018) SX-Ella Stent Danis Effectively Controls Refractory Variceal Bleed in Patients with Acute-onChronic Liver Failure. Dig Dis Sci 63: 493-501. [Crossref]

15. Shao XD, Qi XS, Guo XZ (2016) Esophageal Stent for Refractory Variceal Bleeding: A Systemic Review and Meta-Analysis. Biomed Res Int 2016: 4054513.

16. Marot A, Trépo E, Doerig C, Moreno C, Moradpour D, et al. (2015) Systematic review with meta-analysis: self-expanding metal stents in patients with cirrhosis and severe or refractory oesophageal variceal bleeding. Aliment Pharmacol Ther 42: 1250-1260. [Crossref]

17. Henderson JM (2001) Salvage therapies for refractory variceal hemorrhage. Clin Liver Dis 5: 709-25.

18. Lv Y, Yang Z, Liu L, Li K, He C, et al. (2019) AVB-TIPS Study Group. Early TIPS with covered stents versus standard treatment for acute variceal bleeding in patients with advanced cirrhosis: a randomised controlled trial. Lancet Gastroenterol Hepatol 4: 587-598. [Crossref]

19. Maimone S, Saffioti F, Filomia R, Alibrandi A, Isgrò G, et al. (2019) Predictors of Rebleeding and Mortality Among Patients with Refractory Variceal Bleeding Undergoing Salvage Transjugular Intrahepatic Portosystemic Shunt (TIPS). Dig Dis Sci 64:13351345. [Crossref]

20. Jamwal KD, Padhan RK, Sharma A (2020) Refractory Variceal Bleeding: Approach to Management (Mini Review). World J Gastroenterol Hepatol Endosc 3: 1-3.

Copyright: $\odot 2020$ Jamwal KD. This is an open-access article distributed under the terms of the Creative Commons Attribution License, which permits unrestricted use, distribution, and reproduction in any medium, provided the original author and source are credited. 